Case Report

\title{
Psoas abscess caused by probable extended spectrum beta lactamase (ESBL) producing Escherichia coli: A Case Report
}

\author{
JALU Kumara, GWD Somasiri, DM Mendis, CN Wijesinghe, WD Galagedara, \\ AM Abeygunasekara, S Mendis, NS Chandrasiri
}

Sri Lankan Journal of Infectious Diseases 2021 Vol.11(2):126-130

DOI: http://dx.doi.org/10.4038/sljid.v11i2.8408

\begin{abstract}
Iliopsoas abscess (IPA) is a collection of pus in the iliopsoas muscle. It is rare and usually presents with vague symptoms. IPA can be either primary or secondary. An IPA was diagnosed by computed tomography $(\mathrm{CT})$ in a 64-year-old male with diabetes mellitus and chronic kidney disease (CKD) who presented with a limp. Pus specimen from IPA yielded non-lactose fermenting Escherichia coli which is rarely isolated from IPA. The isolate was sensitive to meropenem and treated with intravenous meropenem for 4 weeks. Source control was achieved by ultrasound scan-guided aspiration of the abscess.
\end{abstract}

Keywords: Iliopsoas abscess, non-lactose fermenting Escherichia coli, extended spectrum beta lactamase, chronic kidney disease

\section{Introduction}

Iliopsoas abscess (IPA) is a rare condition with varied symptomatology which makes the clinical diagnosis difficult. Escherichia coli is the most common facultative anaerobe in the human gastrointestinal tract. ${ }^{1}$ E. coli causing IPA is relatively rare according to the available literature. Early diagnosis and correct management of the patient with IPA would result in a better outcome.

\section{Case report}

A 64-year-old man with diabetes mellitus, chronic kidney disease and ischaemic heart disease presented with a history of right side lower abdominal pain for 3 days. Mild tenderness in the right iliac fossa and limping were detected on examination. He was admitted with similar

Colombo South Teaching Hospital, Kalubowila, Sri Lanka

Address for correspondence: Dr JALU Kumara, No 82/4, St. Jude Mw, Kandy Rd, Dalugama, Kelaniya, Sri Lanka.. Telephone:+0094718129225 E-mail: ja.lasantha@gmail.com

(1D https://orcid.org/0000-0003-2539-6104

Received 29 June 2021 and revised version accepted 24 September 2021.

(c) (7)

This an open-access article distributed under the terms of the Creative Commons Attribution License, which permits unrestricted use, distribution, and reproduction in any medium, provided the original author and source are credited. 
presentation one week previously and was treated with oral ciprofloxacin for 5 days. Urinary tract infection was suspected and he was sent home while awaiting culture results. Urine culture grew $>10^{5}$ non-lactose fermenting (NLF) coliform sensitive to co-amoxiclav, nitrofurantoin and co-trimoxazole.

On admission, abdominal ultrasonography (USS) showed acute pyelonephritis and pelvicalyceal system fullness in the right kidney. Oral co-amoxiclav was begun which was changed to intravenous (IV) cefotaxime on day 1 after admission. CT scan (done at day 4 of admission) showed a right side psoas abscess causing compression of the right ureter and hydronephrosis. Intravenous metronidazole was added to cover a possible mixed infection including anaerobes. USS guided aspiration was performed on day 11 of admission and the aspirate sent for aerobic bacterial culture, staining for acid fast bacilli, GeneXpert ${ }^{\circledR}$ and culture for M. tuberculosis.

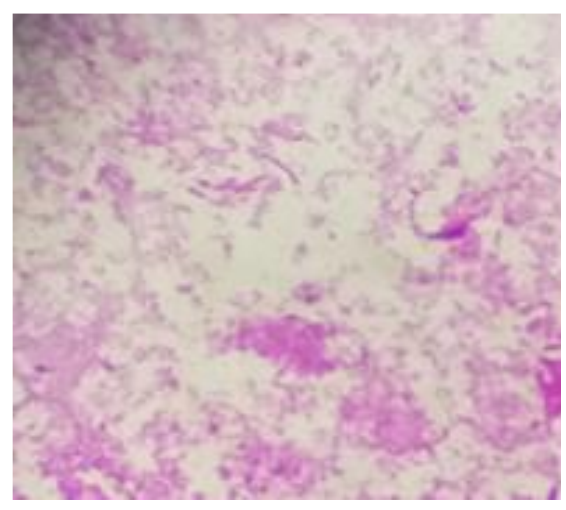

Fig. 1:

Gram stain of the direct smear x100 (pus from psoas abscess)

Gram negative bacilli were observed in the direct smear (Fig. 1) and pus culture yielded a pure growth of a NLF coliform. E. coli was identified with $>99.9 \%$ probability by a commercial identification system (Remel RapID $\left.\mathrm{ONE}^{\mathrm{TM}}\right)$. The isolate was only sensitive to meropenem and co-trimoxazole. Antibiotics were changed to IV meropenem. A repeat USS showed a residual abscess. Blood culture done on day 4 of the admission was negative. On day 17 of admission a pigtail catheter was inserted for continuous drainage of the abscess. The pus specimen from the repeat aspiration grew a scanty growth of the NLF coliform which was similar to the first isolate in morphology and antibiotic sensitivity. Rigid cystoscopy was done and double $\mathrm{J}$ stent was inserted into the right ureter 22 days after admission. The patient was treated with meropenem for 4 weeks and complete resolution confirmed with a repeat radiological assessment. The pigtail catheter was removed and he was sent home on oral co-amoxiclav for one week. Serum creatinine which was $631 \mu \mathrm{mol} / \mathrm{L}$ initially, reduced to $336 \mu \mathrm{mol} / \mathrm{L}$ with the clearance of the infection without haemodialysis. Pus sent for GeneXpert ${ }^{\circledR}$ and culture for $M$. tuberculosis were negative. During the follow up ( 2 weeks after discharge) he was clinically well, and double $\mathbf{J}$ stent was removed according to the surgical protocol. The timeline of the illness is given in Figure 2. 


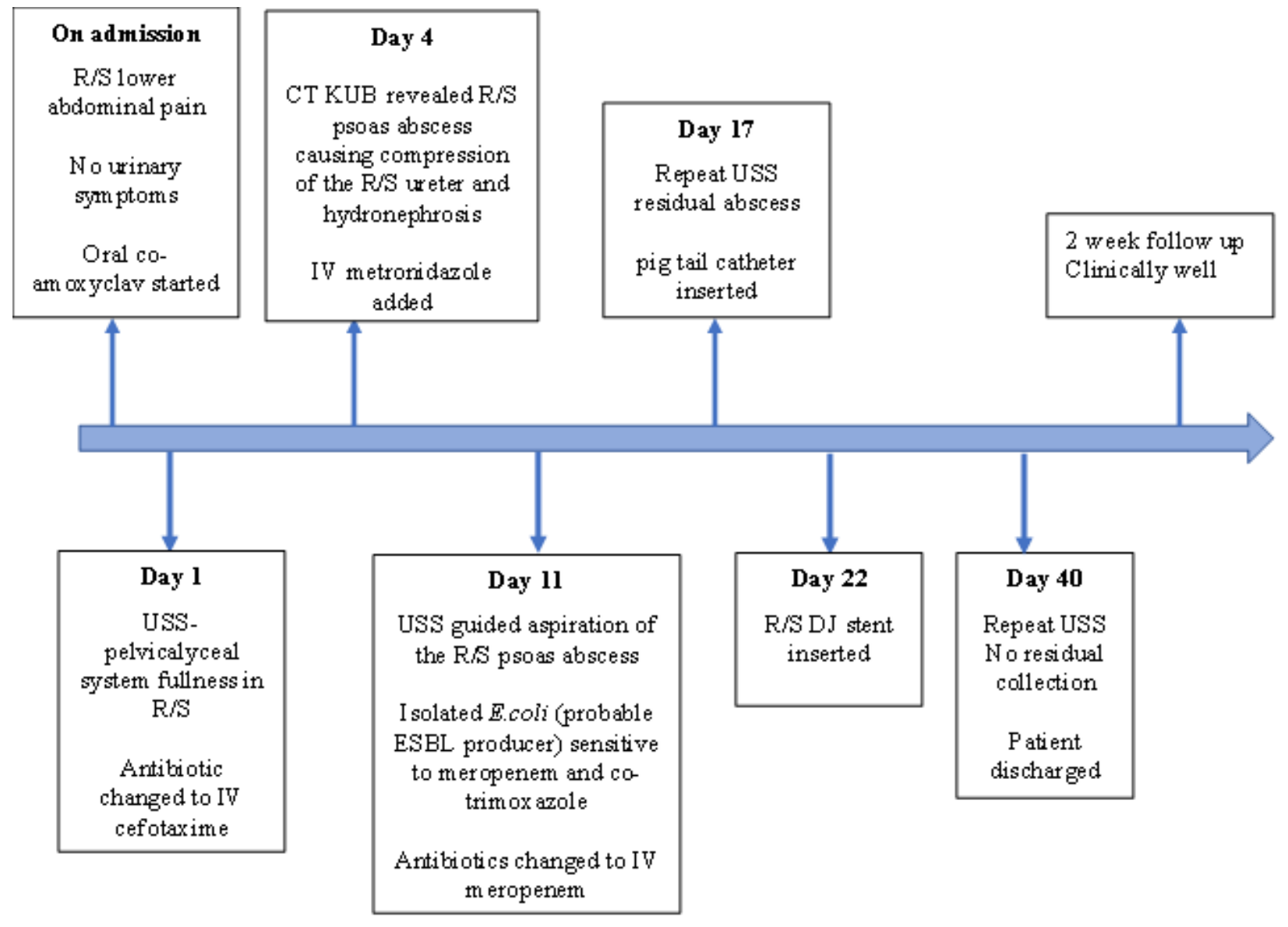

Fig 2: Timeline of the illness

\section{Discussion}

IPA is an uncommon condition with vague presentation. IPA can be primary or secondary. Risk factors include renal failure, diabetes mellitus, immunosuppression, HIV and intravenous drug use. It may occur secondary to infections in the gastrointestinal, genitourinary tract or musculoskeletal system. In the United States of America (USA), 85\% cases of IPA occur as secondary to infections in skeletal (46\%), gastrointestinal (28\%), cardiovascular (14\%) and genitourinary $(3.7 \%)$ systems. $^{2}$

Mynter in 1881 described a classic triad of fever, flank pain and limp in IPA. In a study done in Hong Kong, among 37 patients with a psoas abscess, none presented with the classic triad. Commonest complains were back/thigh/hip pain (43\%), fever (41\%), limited hip movement $(22 \%)$ and abdominal pain $(14 \%){ }^{3}$

Imaging is the mainstay of diagnosis. In a USA study, diagnosis was made by CT in $71 \%$, magnetic resonance imaging (MRI) in 26\%, white blood cellular nuclear scan in $2 \%$ and USS in $1 \%{ }^{3}$ MRI is considered superior to CT in diagnosis of IPA. From a Sri Lankan cohort of patients suspected of tuberculosis who underwent MRI, $23 \%$ had psoas abscess. ${ }^{4}$ 
Pathogens isolated from primary or secondary IPA may vary with underlying aetiology and geographical location. The most common organism found in both primary and secondary IPA in USA is Staphylococcus aureus..$^{5}$ An Indian study showed Mycobacterium tuberculosis as the commonest cause of IPA. ${ }^{6}$ Pathogens causing primary IPA include S. aureus, coagulase negative staphylococci (CoNS), M. tuberculosis and occasionally E. coli. In secondary IPA, in addition to these, Klebsiella spp., Enterococcus spp., Proteus spp., Bacteroides spp., Peptostreptococcus spp., Clostridium spp., and Salmonella Enteritidis have been reported. ${ }^{7}$ A study in Hong Kong reported that $85.1 \%$ of the isolates from IPA were monomicrobial and the isolated pathogens were Staphylococcus aureus (29.6\%), M. tuberculosis $(26 \%)$ and Enterobacteriaceae $(21.7 \%){ }^{3}$ In USA, Staphylococcus spp. (69\%) was the predominant isolate followed by E. coli (16\%), Bacteroides spp. (12\%), Prevotella spp. (10\%), and Fusobacterium spp. (8\%). ${ }^{2}$ As for causative organisms of IPA in Sri Lanka, Badurdeen et al described a renal transplant recipient who developed bilateral psoas abscesses due to $S$. aureus. ${ }^{8}$ A study from Greece reported only 5 cases of IPA within a 10 year period. Among those, only one isolate was found to be $E$. coli from mixed growth ${ }^{9}$. In Sri Lanka, studies related to causative agents of IPA are rare with none reporting an ESBL producing E. coli. ${ }^{10,11,12}$ Isolation of ESBL producing E. coli which is nonlactose fermenting from IPA was not reported in the literature.

Principles of management include source control either by percutaneous aspiration or open surgery with appropriate antimicrobial therapy.

Our patient had presented with urinary symptoms previously. He complained only of abdominal pain and limited hip movements in this presentation. Accurate early diagnosis was possible only through USS which revealed acute pyelonephritis followed by CT scan to diagnose the IPA. This emphasizes the importance of proper imaging in the early diagnosis of IPA.

Previous undetected bacteriaemia due to E. coli secondary to urinary tract infection may have been the cause of a secondary psoas abscess in our patient. Organism identification was by Remel Rap ID ONE ${ }^{\mathrm{TM}}$ with a probability of more than $99.9 \%$. According to Clinical \& Laboratory Standards Institute (CLSI) recommendation for testing, this isolate was a probable extended spectrum beta lactamase (ESBL) producer. ${ }^{13}$ It is possible that the isolate previously isolated from the patient's urine acquired the resistance demonstrated by the isolate from the abscess. Repeated prescriptions for urinary tract infections may have led to selection of ESBL forming E. coli. ${ }^{14}$ The clinical significance of slow or non-lactose fermenting E. coli $(10 \%)$ is unknown. ${ }^{15}$ The patient was discharged on oral co-amoxiclav.

Without the diagnosis of IPA, it is possible that this patient would have received a short course of antibiotics before discharge and may have presented with life-threatening Gram-negative sepsis. However, a multidisciplinary approach with prompt radiological and microbiological investigations resulted in a favourable outcome.

\section{Declarations}

Conflict of Interest: None declared

Acknowledgments - We thank the patient and family for consenting for the publication of the case details

Ethics statement and consent for publication: Written consent was taken from the patient

Author contributions - J.A.L.U. Kumara, G.W.D. Somasiri, D.M. Mendis, C.N. Wijesinghe, W.D..Galagedara- participate in writing the manuscript ; A.M. Abeygunasekera, S. Mendis, N.S. Chandrasiri - editing the manuscript 


\section{References}

1. John EB, Raphael D, Martin JB. (9 $9^{\text {th }}$ edition) Mandell, Douglas, and Bennett's Principles and Practice of Infectious Diseases. Philadelphia, PA: Elsevier/Saunders, 2019: pp 2669-2684.

2. Ouellette L, Hamati M, Flannigan M et al. Epidemiology of and risk factors for iliopsoas abscess in a large community-based study. American Journal of Emergency Medicine 2019; 37(1):158-159. doi: 10.1016/j.ajem.2018.05.021

3. Wong OF, Ho PL, Lam SK. Retrospective review of clinical presentations, microbiology, and outcomes of patients with psoas abscess. Hong Kong Medical Journal 2013; 19(5):416-423. doi: $10.12809 / \mathrm{hkmj} 133793$.

4. Pallewatte AS, Wickramasinghe NA. Magnetic resonance imaging findings of patients with suspected tuberculosis from a tertiary care centre in Sri Lanka. Ceylon Medical Journal 2012: 61:185-188 doi: http://doi.org/10.4038/cmj.v61i4.8387.

5. Shields D, Robinson P, Crowley TP. Iliopsoas abscess - A review and update on the literature. International Journal of Surgery 2012; 10(9):466-469. doi: 10.1016/j.ijsu.2012.08.016

6. Rodrigues J, Iyyadurai, R, Sathyendra S et al. Clinical presentation, etiology, management, and outcomes of iliopsoas abscess from a tertiary care center in South India. Journal of Family Medicine and Primary Care 2017; 6(4):836-839. doi: 10.4103/jfmpc.jfmpc_19_17

7. Lipsett PA. Psoas abscess. in: Schlossberg, D. (Ed.), Clinical Infectious Disease. Cambridge University Press Cambridge, 2008: pp.495-502. doi: https://doi.org/10.1017/CBO9780511722240.073

8. Badurdeen AS, Rajakrishna PN, Herath HMNJ et al. Bilateral psoas abscess in a renal transplant patient. Ceylon Medical Journal 2012; 57:175-176 doi: 10.4038/cmj.v57i4.5090

9. Charalampopoulos A, Macheras A, Charalabopoulos A et al. Iliopsoas abscesses: diagnostic, aetiologic and therapeutic approach in five patients with a literature review. Scandinavian Journal of Gastroenterology 2009; 44(5):594-599. doi: 10.1080/00365520902745054

10. Corea EM., Merritt AJ, Ler YH et al. Sri Lankan national melioidosis surveillance program uncovers a nationwide distribution of invasive melioidosis. The American journal of tropical medicine and hygiene 2016; 94(2):292. doi: 10.4269/ajtmh.15-0567

11. Karunarathna AKTM., Mendis SA, Perera WPDP et al. A case report of melioidosis complicated by infective sacroiliitis in Sri Lanka. Tropical diseases, Travel medicine and Vaccines 2018; 4(1):1-6. doi:org/10.1186/s40794-018-0073-5

12. Duminda HKKT, Lamahewage AK, Liyange A et al. Iliopsoas abscess due to methicillin resistant Staphylococcus aureus in a 26 day old neonate. Sri Lanka Journal of Child Health 2015 44(3):16768. doi: http://doi.org/10.4038/sljch.v44i3.8017

13. Performance Standards for Antimicrobial Susceptibility Testing, $31^{\text {st }}$ Edition, CLSI guideline, M100. Wayne, PA: Clinical and Laboratory Standards Institute; 2021.

Available at https://clsi.org/standards/products/free-resources/access-our-free-resources/

14. Hillier S, Roberts Z, Dunstan F et al. Prior antibiotics and risk of antibiotic-resistant communityacquired urinary tract infection: a case-control study. Journal of Antimicrobial Chemotherapy 2007; 60(1):92-99. doi: doi: 10.1093/jac/dkm141

15. Yaratha G, Perloff S, Changala K. Lactose vs non-lactose fermenting E. coli: Epidemiology, Clinical Outcomes, and Resistance. Open Forum Infectious Diseases 2017; 4(1):S589-S590.

doi: https://doi.org/10.1093/ofid/ofx163.1546. 